\title{
Efectos diversos en calidad y costos de los programas de cuidados coordinados
}

Diverse effects of care coordination programs on quality of care and health expenditures

Peikes, D y col. JAMA. 2009;301(6):603-618

\section{Objetivo}

Determinar si los programas de cuidado coordinado (PCC) reducen hospitalizaciones, gastos y/o mejoran la calidad del cuidado en beneficiarios con enfermedades crónicas.

\section{Diseño y lugar}

Ensayos clínicos aleatorizados, controlados y abiertos, en 15 PCC del Medicare (EE.UU.). Fueron llevados a cabo en localidades y ámbitos diversos (cinco compañías comerciales, tres hospitales comunitarios, tres centros académicos, un sistema de atención integrado y tres centros de retiro o cuidados de tercer nivel) sobre una población de beneficiarios de Medicare con al menos una enfermedad crónica. En su mayoría, fueron pacientes con enfermedad coronaria, insuficiencia cardíaca, diabetes y enfermedad pulmonar obstructiva crónica, además de neoplasias, accidente cerebrovascular, depresión y demencia.

\section{Medición de resultados principales}

Utilización hospitalaria y gastos de Medicare, así como medidas de proceso y resultado de la calidad del cuidado. Cada PCC fue evaluado independientemente, de acuerdo a protocolos locales consensuados previamente sobre la población elegible y las intervenciones determinadas.

\section{Resultados principales}

Los pacientes enrolados tenían un nivel sociocultural y generaban un gasto mayor que el promedio de los de Medicare, mien- tras que su proporción de hispanos fue menor que el promedio. La media de seguimiento en cada PCC varió entre 18 y 38 meses y varios programas tuvieron bajo poder para detectar diferencias de costos y/o hospitalizaciones.

La mayoría de los 15 programas no mostró diferencias significativas en la tasa de hospitalización, excepto dos de ellos: 1) el de Mercy, con 0,168 menos hospitalizaciones por persona/año (IC90\% $-0,283$ a $-0,054)$ lo que representa un $17 \%$ menos que el grupo control $(p=0,02) ; 2)$ el de Charlestown, con 0,118 más hospitalizaciones (IC90\% 0,025 a $0,210)$ un $19 \%$ más que el control $(p=0,04)$.

Ninguno de los PCC generó ahorros netos, pero sí mejoras en algunos de los indicadores de calidad de los cuidados.

\section{Conclusiones}

Los PCC sin un fuerte componente de transición del cuidado difícilmente puedan generar ahorros netos a Medicare. Los programas con un significativo contacto persona a persona que apunten a pacientes moderada o severamente enfermos pueden ser neutrales en costos y mejorar algunos aspectos del cuidado.

Palabras claves: coordinación de cuidados, internaciones, calidad de cuidado, gastos en salud, ensayo clínico aleatorizado.

Key words: care coordination, hospitalization, quality of care, health care expenditures, ranKey words: care coordin
domized controlled trial.

Fuente de financiamiento: Center for Medicare and Medicaid Services. Los autores reportan no tener conflictos de interés.

\section{Comentario}

El concepto fundamental que transmite este importante trabajo es que no hay soluciones mágicas, ya que no cualquier PCC de pacientes con enfermedades crónicas generará automáticamente una disminución de internaciones y/o de costos. Entre sus virtudes, mencionamos el haber evaluado 15 programas distintos y en medios diversos (todos de EEUU) con objetivos e intervenciones definidas localmente por cada institución, con un período de seguimiento considerable, y con un diseño aleatorizado y controlado. Más allá de que los resultados globales fueron negativos, es importante tener en cuenta dos detalles importantes. 1) Seis de los 15 estudios tuvieron un bajo poder ${ }^{*}$ para detectar diferencia de hospitalizaciones y cinco para detectar diferencias en costos; 2) las disminuciones de internaciones y costos esperadas eran exigentes (un tamaño del efecto de 20\%). Si bien esto fue estimado en función de estudios previos, la mayor oportunidad de mejora se ve en sistemas de atención totalmente desarticulados o con mala calidad de atención previa. Por el contrario y como es lógico suponer, en sistemas con mejores resultados basales, las diferencias observadas pueden ser menores. En este sentido vale destacar que cinco de los estudios tuvieron una reducción de más del $5 \%$ en las internaciones respecto al grupo control. Por eso pensamos que una diferencia real más pequeña a la esperada pudo no haber sido detectada por este motivo.
Los dos programas con mejores resultados (Mercy y HQP) tenían un promedio de contacto cara a cara con los pacientes mucho mayor que casi todos los que fracasaron. A diferencia de la mayoría del resto de los programas, los coordinadores del cuidado de Mercy y HQP trabajaban en contacto estrecho con los hospitales locales y también con los médicos tratantes. ¿Por qué son importantes estas consideraciones? En general, la evidencia acerca de la efectividad de los PCC es favorable ${ }^{1,2}$. Sin embargo, hay gran heterogeneidad de intervenciones y de documentación de su efectividad. Actualmente existe experiencia y evidencia acerca de qué características deberían tener los programas para favorecer su éxito ${ }^{3}$. Entre estas características se destaca la necesidad de que exista contacto personal con los pacientes y que la atención sea integrada con los centros de referencia y médicos tratantes.

\section{Conclusiones del comentador}

En vez de pensar en ahorrar costos como principal objetivo deberíamos discutir qué intervenciones factibles son las que aportan más valor para la sociedad ${ }^{4}$. Por otro lado y para Latinoamérica en particular, el éxito -que consideramos deseable y posible- depende de lograr una atención integrada en el contexto de un cambio de paradigma del sistema de atención en salud 5 .

Gaston Perman [ Programas Médicos, Hospital Italiano de Buenos Aires. Gaston.perman@hospitalitaliano.org.ar ]

Ver glosario*

Recibido el 15/08/09 y aceptado el 01/10/09

Perman G. Efectos diversos en calidad y costos de los programas de cuidados coordinados. Evid. Act. Pract. Ambul. 12(4). 133. Oct-Dic. 2009. Comentado de: Peikes, D y col. Effects of Care Coordination on Hospitalization, Quality of Care, and Health Care Expenditures Among Medicare Beneficiaries: 15 Randomized Trials. JAMA. 2009;301(6):603-618 19211468. Disponible en URL: http://jama.amaassn.org/cgi/content/full/301/6/603

\section{Referencias}

1. Roccaforte R y col. Effectiveness of comprehensive disease management programmes in improving clinical outcomes in heart failure patients. A meta-analysis. Eur J Heart Fail. 2005 Dec;7(7):1133-44. Epub 2005 Sep 29. 2. Clark A y col. Meta-Analysis: Secondary Prevention Programs for patients with Coronary Artery Disease. Ann Intern Med. 2005; 143:659-672.

3. Sochalski J y col. What works in chronic care management: the case of heart failure. Health Aff (Millwood). 2009 Jan-Feb;28(1):179-89.

4. Cohen J y col. Does Preventive Care Save Money? Health Economics and the Presidential Candidates. NEJM 358;7:661-663.

5. González Bernaldo de Quirós F y col. La gestión del cuidado de los pacientes con enfermedades crónicas. ¿Hacia el rediseño de los sistemas de salud? Rev. Hosp. Ital. B.Aires. 2008;28(1):5-11. 